Journal of Computer Science 7 (5): 678-682, 2011

ISSN 1549-3636

(C) 2011 Science Publications

\title{
Varying Overhead Ad Hoc on Demand Vector Routing in Highly Mobile Ad Hoc Network
}

\author{
${ }^{1} \mathrm{~V}$. Balaji and ${ }^{2} \mathrm{~V}$. Duraisamy \\ ${ }^{1}$ Department of Electronics and Communication Engineering, \\ Hindusthan College of Engineering and Technology, \\ ${ }^{2}$ Hindusthan College of Engineering and Technology, \\ Coimbatore, Tamilnadu, 641 032, India
}

\begin{abstract}
Problem statement: An inherent feature of mobile ad hoc networks is the frequent change of network topology leading to stability and reliability problems of the network. Highly dynamic and dense network have to maintain acceptable level of service to data packets and limit the network control overheads. This capability is closely related as how quickly the network protocol control overhead is managed as a function of increased link changes. Dynamically limiting the routing control overheads based on the network topology improves the throughput of the network. Approach: In this study we propose Varying Overhead - Ad hoc on Demand Vector routing protocol (VO-AODV) for highly dynamic mobile Ad hoc network. The VO-AODV routing protocol proposed dynamically modifies the active route time based on the network topology. Results and Conclusion: Simulation results prove that the proposed model decreases the control overheads without decreasing the QOS of the network.
\end{abstract}

Key words: Mobile Ad Hoc Network (MANET), Ad Hoc on Demand Distance Vector (AODV), Dynamic Source Routing (DSR), Distance Sequenced Distance Vector (DSDV), Optimized Link State Routing (OLSR), Fish Eye State Routing (FSR), Active Route Timeout (ART), route error, associativity based routing

\section{INTRODUCTION}

A Mobile Ad Hoc Network (MANET) is a group of wireless mobile nodes dynamically forming a network for data transfer without any pre existing infrastructure or centralized administration. Because of its infrastructure-less nature it is easy to deploy and instantly finds extensive applications in military, business and academia (Kalaavathi and Duraiswamy, 2008). In a MANET each and every individual node has the additional task of a router by which it can forward packets between two or more nodes. As the network is dynamic the challenge in the network is to maintain the correct routes as the topology changes continuously. The routing protocol in MANET should be capable of adjusting between high mobility, low bandwidth to low mobility, high bandwidth scenario.

As routing plays an important role for the reliability of an Ad hoc network many routing methodologies have been proposed and these protocols can be broadly classified into proactive and reactive routing protocols. Proactive routing protocols discover routes for every pair of nodes by continuously updating the routing tables at fixed time intervals irrespective of data traffic between source and destination. A route is available immediately when communication is to be established between a source and destination. Proactive routing protocol based wireless networks have additional overheads in the network due to constant updation of route traffic but end to end delay is minimized. On the other hand reactive routing protocols establish a route to destination only when there is a requirement. Though the network control packet overheads are reduced in reactive routing protocol, the end to end delay increases due to the route discovery process (Alandzi and Quintero, 2007). Popular reactive routing protocol include Ad Hoc on Demand Distance Vector (AODV) routing, Dynamic Source Routing (DSR) Associativity Based Routing (ABR) (Toh, 1997) and some of the pro active routing protocols include Distance Sequenced Distance Vector(DSDV) routing (Perkins and Bhagwat., 1994), Optimized Link State Routing (OLSR) and Fish Eye State Routing (FSR) (Peri et al., 2000).

Corresponding Author: V. Balaji, Department of Electronics and Communication Engineering Hindusthan, College of Engineering and Technology, Coimbatore, Tamilnadu, 641 032, India 
Even though reactive protocols discover routes as and when required they still keep some of the route state information for a specific period of time. This reduces the overheads of route establishment as the route that is remembered can be used again if it is still valid in the near future. The duration of time for which the route state is maintained after communication between source and destination is complete plays a important role in reducing network overheads (Richard et al., 2005). However Ad hoc networks are highly mobile and the speed at which the nodes move about the network make it difficult to fix a specific time value for which the route state information is to be maintained. In AODV the route state information is maintained for a time period mentioned in the Active Route Timeout (ART) whereas in DSR timeout is maintained in the Route Cache Timeout.

In this study we investigate the performance of a highly mobile network using Adhoc On Demand Distance Vector (AODV) routing protocol under different active route timeout and propose an enhanced routing algorithm Varying Overhead Adhoc On Demand Distance Vector (VO-AODV) routing protocol. The new protocol is modeled based on how fast the link change occurs and optimizes the protocol overheads to a minimum.

This study is organized into the following sections. Section 2 introduces us to the AODV Routing protocol, Section 3 describes the proposed VO-AODV protocol, Section 4 describes the experimental setup with the results obtained and Section 5 discusses the obtained results.

Ad hoc on demand distance vector routing protocol: AODV is an enhancement of the Bellmann Ford Distance Vector algorithm to work in a wireless environment. Since it is a reactive routing protocol, a route to destination is discovered only when a nodes wants to send a packet to that destination. Sequence numbers are used to ensure the freshness of the route and to provide loop free routing. The routing table contains:

- Destination

- Next hop

- Number of hops to reach destination

- Destination sequence number

- Active neighbors for this route

- Route table entry expiration time

AODV uses a series of control messages to determine and maintain routes. The control messages used by this protocol are Route request (RREQ), Route Reply (RREP), Route Error (RERR) and Hello messages. When a node wants to send data to a destination node and if a route is not available it floods the network with a RREQ. When a source sends an RREQ it increments the request ID and each RREQ from a source is identified uniquely using the source address, request ID pair. The intermediate node will accept the first unique RREQ and discard all others if it has the same pair of parameters. The received RREQ will then be broadcasted after increasing the hop count if the node is not the destination or unicasts a RREP if it is the destination or if it has a route entry whose sequence greater than or equal to that of RREQ. In AODV all nodes monitor their neighbor and whenever a node in a active route is not available, a RERR is generated to notify the nodes on either side of the link. Nodes use HELLO messages to continuously monitor the neighbor nodes.

Various enhancements have been proposed using AODV protocol. An improved AODV for load balancing have been proposed (Rani and Dave, 2007) for route discovery using aggregate interface queue length. AODV-BRL was proposed (Yujun and Lincheng, 2010) to increase the adaptation of routing protocols to topology changes by enhancing AODV-BR.

Proposed AODV model: VO-AODV: Various routing algorithm modification have been proposed (Natsheh and Buragga, 2010) In an Ad hoc network the throughput is affected by the speed at which the nodes move. Throughput also depends on the utilization of route cache (Elfaki et al., 2011). As the nodes move over the network the topology also changes continuously which affects the overall network performance and is dependent on the routing protocol being used. In theory reactive routing protocol should perform better than other routing protocols in a high mobility network. The route life represented by Active Route Timeout (ART) in AODV routing protocol plays an important role in managing fast change in topology. Typical values of ART is 3 seconds which is not justified as the ART value is network dependent. In a static network the ART value can be arbitrarily high and in very fast moving node (assuming the node is a car) ART values has to be less than 1 second.

To overcome this problem in reactive routing protocols, the Varying Overhead-AODV is proposed. The additional steps involved over the routing overhead in each node are:

- At time $t_{1}$, for a duration $n$, compute power from all nodes $s_{i}$ transmitting data to node $D$. Let the power 
computed from each node be $\mathrm{P}_{\mathrm{i}}$. The total power is from all nodes for the duration is $\sum \mathrm{P}_{\mathrm{i}}$

- At time $t_{2}$, for a duration $n$, compute power received from the same sources. Let the power computed for each node be $P_{j}$ from one packet received. The total power computed is given by $\sum \mathrm{P}_{\mathrm{j}}$

- Compute $\mathrm{x}=\sum \mathrm{P}_{\mathrm{j}}-\sum \mathrm{P}_{\mathrm{i}}$

- If $\mathrm{x}$ is positive increase ART from reference value by $\mathrm{x} / \mathrm{P}_{\text {imax }}$

- If $x$ is negative decrease ART from reference value by $\mathrm{x} / \mathrm{P}_{\text {imax }}$ provided the computed value does not go below minimum set threshold value

Explanation: We follow an equal opportunity approach. The distance between a source and destination can be computed using the transmitter power output, transmitter antenna gain, receiver sensitivity, receiver antenna gain and free space loss.

At two instances $t_{i}+n$ and $t_{2}+n$ we compute the power from each of the source nodes transmitting data to the receiver node. If most of the sources are close to each other, the probability of routes going stale is lower and hence we can increase the ART. Since we are measuring power at two instance of time, it is possible to predict whether the sources are moving towards the receiver node or away from it. The ART is changed accordingly based on the number of sources moving towards the destination or away from it.

\section{MATERIALS AND METHODS}

We implemented our hypotheses using OPNET. Opnet is a popular network simulator and avoids the need for wireless test bed. Various types of wireless nodes, routers and routing protocols are built into the simulator for fast setup and simulation. Opnet provides methods to create nodes, routers and trajectories for nodes. Twenty nodes with random mobility and speeds were implemented in a 490000 sq.m network. Each node has a transmit power of 0.005 watts. Each node randomly transmits raw unformatted data to every other node in the network. Simulation in each scenario was carried out for 600 second. Simulations were carried out with ART $=2$ second, 4 second, 6 second and our proposed method. The obtained results are shown in Fig. 1-4.

\section{RESULTS AND DISCUSSION}

From Fig. 1 it is seen that for a high mobility scenario Active route timeout of 2 second (Blue graph) gives the best throughput. The proposed routing algorithm (Yellow graph) has an initial settling period after which the throughput is comparable with ART $=2$ second. The throughput almost remains the same for the other three scenarios of ART values 3,4 and 6 .

Figure 2-4 gives the cached replies, routing traffic received and routing traffic sent. As the ART increases the output obtained are on expected lines.

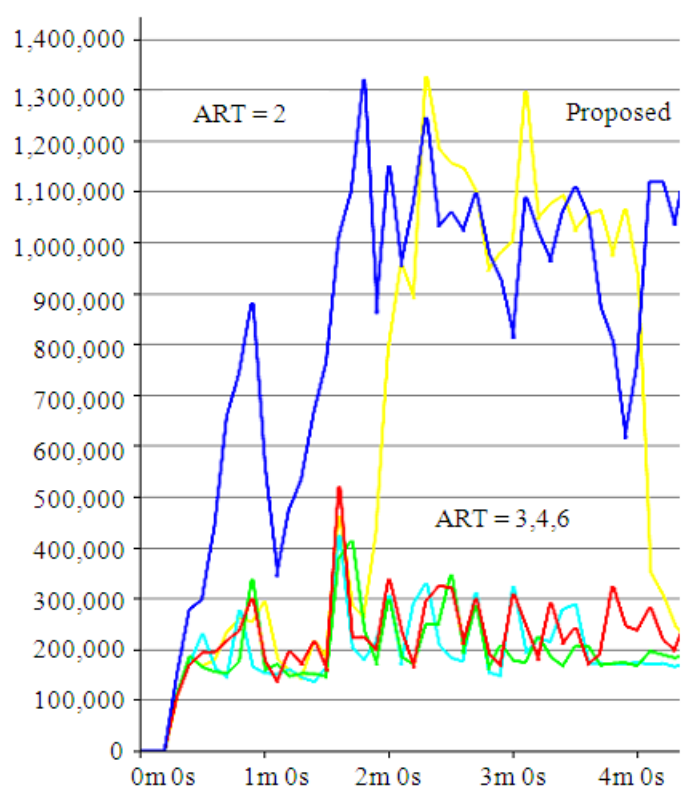

Fig. 1: The throughput obtained for various ART values and the proposed VO-AODV algorithm

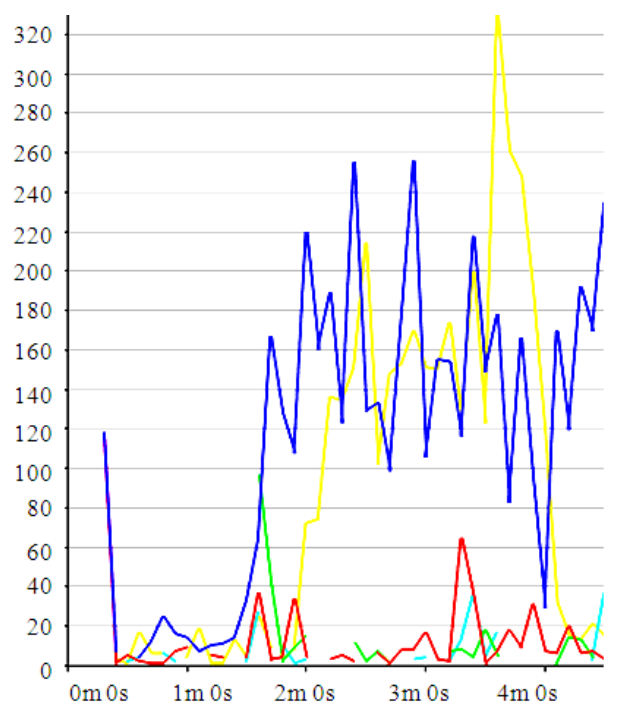

Fig. 2: Total Cached replies sent 


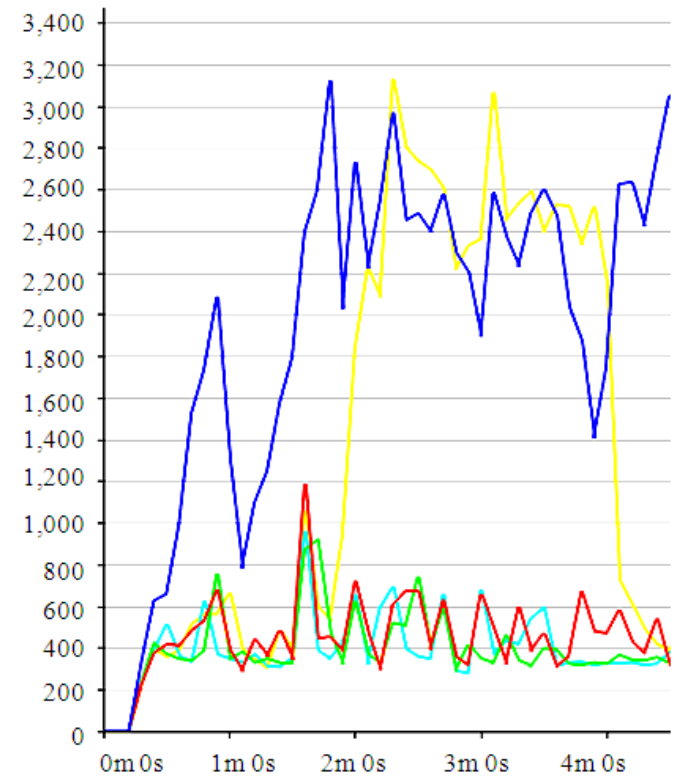

Fig. 3: Routing traffic received

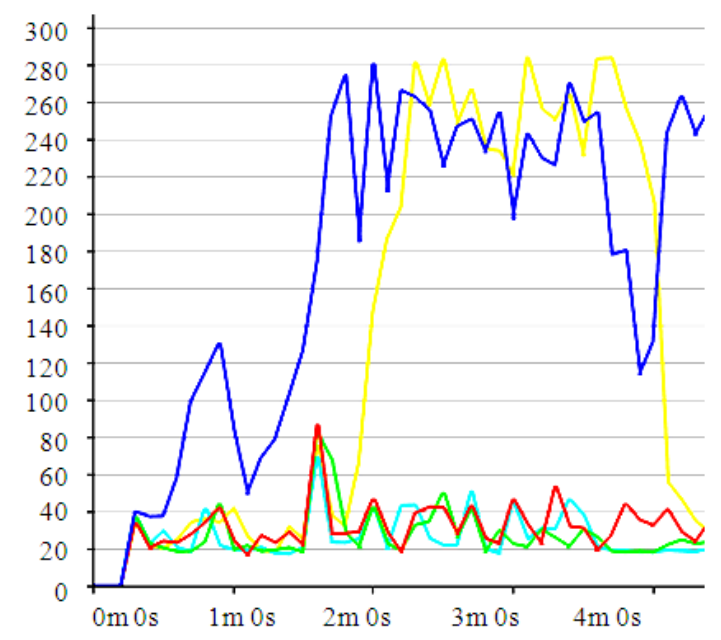

Fig. 4: Routing traffic sent

However it is to be noted that for ART values of 3 and 4 seconds the obtained parameters are same. However this needs to be investigated for other mobility scenarios.

\section{CONCLUSION}

In this study we proposed a novel routing algorithm which is an extension of the Ad hoc on demand distance vector routing protocol. The proposed method identifies how the network topology changes based on a node and its next one hop nodes. Computation of power of each node within one hop distance of the destination node is computed periodically and used to identify whether nodes are converging or diverging. Based on the convergence or divergence of nodes we proposed an algorithm to modify the Active Route Timeout.

Results obtained from the proposed method did not decrease the throughput compared to the standard value of ART with considerable decrease in network overheads.

\section{REFERENCES}

Alandzi, V. and A. Quintero, 2007. Proximity aware routing in Ad Hoc Networks. J. Comput. Sci., 3: 533-539. DOI: $10.3844 /$ jcssp.2007.533.539

Elfaki, M.A., H. Ibrahim, A. Mamat and M. Othman, 2011. Collaborative caching architecture for continuous query in mobile database. Am. J. Econ. Busi. Admin., 3: 33-39. DOI: 10.3844/ajebasp.2011.33.39

Kalaavathi, B. and K. Duraiswamy, 2008. Ant colony based node disjoint hybrid multi-path routing for mobile ad hoc network. J. Comput. Sci., 4: 80-86. DOI: $10.3844 /$ jcssp.2008.80.86

Natsheh, E. and K. Buragga, 2010. Density based routing algorithm for spare/dense topologies in wireless mobile ad-hoc networks. Am. J. Eng. Applied Sci., 3: 312-319. DOI: 10.3844/ajeassp.2010.312.319

Peri, G., M. Gerla and T.-W. Chen, 2000. Fisheye state routing: A routing scheme for Ad Hoc Wireless Networks. Proceedings of the International Conference on Communications, (ICC'00), IEEE Xplore, USA., pp: 70-74. DOI: 10.1109/ICC.2000.853066

Perkins, C.E. and P. Bhagwat, 1994. Highly Dynamic Destination-Sequenced Distance-Vector Routing (DSDV) for Mobile Computers. Proceedings of the conference on Communications architectures, protocols and applications, (SIGCOMM'94), ACM New York, NY, USA., pp: 234-244. DOI: $10.1145 / 190314.190336$

Rani, A. and M. Dave, 2007. Performance evaluation of modified aodv for load balancing. J. Comput. Sci., 3: 863-868. DOI: $10.3844 /$ jcssp.2007.863.868

Richard, C., C. Perkins and C. Westphal, 2005. Defining an Optimal Active Route Timeout for the AODV Routing. Nokia Research Center. http://www.mendeley.com/research/definingoptimal-active-route-timeout-aodv-routing/ 
Toh, C.K., 1997. Associativity-based routing for ad hoc mobile networks. Wireless Personal Communications. Associativity-based routing for ad hoc mobile networks. Wireless Personal Communi., 4: 103-139.
Yujun, L. and H. Lincheng, 2010. The research on an AODV-BRL to increase reliability and reduce routing overhead in MANET. Proceedings of the International Conference on Computer Application and System Modeling, Oct. 22-24, IEEE Xplore, Taiyuan, pp: 526-530. DOI: 10.1109/ICCASM.2010.5622386 